\title{
Il parere dei pazienti sui prodotti ipoproteici nella terapia dietetica dell'insufficienza renale cronica
}

\author{
Anna Laura Fantuzzi ${ }^{1}$, Francesca Lugli², Rossella Giannini ${ }^{1}$ \\ ${ }^{1}$ Unità Operativa Dipartimentale di Scienza dell'Alimentazione e Dietetica, Nuovo Ospedale Civile S. Agostino- \\ Estense, Azienda Unità Sanitaria Locale, Modena \\ ${ }^{2}$ Dietista, Libero-professionista, Reggio Emilia
}

\begin{abstract}
THE OPINION OF PATIENTS WITH CHRONIC RENAL DISEASE ON LOW-PROTEIN FOODS
Abstract. Nutritional support of the patient with Chronic Renal Failure (CRF) in conservative treatment allows to prevent/control the main metabolic disease as well as to achieve and/or maintain a satisfactory nutritional status postponing replacement therapy (dialysis or transplantation).

When the low-protein diet requires the introduction of special low-protein foods, the dietitian must maintain a particularly close monitoring. This study evaluated the satisfaction with low-protein foods in a sample of 100 patients with CRF related to our Unit of Dietetics in the years 2009-2012, and compared it with that found in a similar study carried out in 2008-2009. The median age of the subjects involved was 65 years (range 17-88 years), with a predominance of males $(57 \%)$. Overall, $88 \%$ of the patients were treated conservatively and $12 \%$ with substitution treatment $(10 \%$ with dialysis and $2 \%$ with transplantation). All patients were treated with a low-protein diet with $0.6 \mathrm{or} 0.8 \mathrm{~g}$ of protein/ $\mathrm{kg}$ ideal body weight/day. Only $6 \%$ of patients defined as "nothing" or "poor" the replaceability of the normal products with the low-protein products. Compared to the previous study, we found an increase in the consumption of bread and substitutes associated with greater liking. Despite the variety and quality of low-protein products has sharply increased over the years, their inclusion in the dietary pattern is one of the factors that most influences the concordance to the dietary treatment.

In relation to the needs of our users, we added to the study a revision of the regional regulations regarding the provision of low-protein products, as these showed a marked heterogeneity across the country.
\end{abstract}

Key words: Low-protein diet, Low-protein foods, Chronic renal disease, Cross-sectional study

Conflict of interest: None.

Financial support: None.

Accettato: 7 Novembre 2014

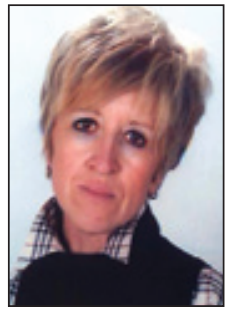

Anna Laura Fantuzzi

\section{Introduzione}

L'alimentazione ha un ruolo cruciale in tutte le fasi evolutive dell'Insufficienza Renale Cronica (IRC) (1-2). Durante la fase conservativa, il trattamento dietetico contribuisce a controllare le principali alterazioni (iperazotemia, acidosi e iperparatiroidismo) e posticipa il trattamento sostitutivo (dialisi o trapianto) (3-6). Grazie alla dimostrata efficacia nel ritardare l'ingresso in dialisi, il trattamento dietetico consente inoltre un risparmio per il Sistema Sanitario Nazionale (3). Il trattamento dietetico effettuato da un dietista esperto migliora la qualità di vita dei pazienti con IRC, aumenta l'efficacia della terapia medica e riduce i costi assistenziali (7-12).

\section{Dieta ipoproteica: effetto sulla "morte renale"}

Una metanalisi Cochrane di 10 studi randomizzati controllati ha dimostrato che la dieta ipoproteica riduce del $31 \%$ l'occorrenza di "morte renale" (morte del paziente, ingresso in dialisi o trapianto) in pazienti con IRC ad eziologia non diabetica. La frequenza di morte renale era $17 \%$ nel gruppo di controllo e $11 \%$ in quello trattato con dieta ipoproteica, corrispondente a una riduzione assoluta del rischio di morte renale pari al $6 \%$ e a un numero di pazienti da trattare in un anno per prevenire una morte renale pari a 18 (3). Uno studio randomizzato controllato effettuato presso lo Steno Diabetes Center ha similmente dimostrato una riduzione della "morte renale" nei pazienti con diabete di tipo 1 e nefropatia diabetica progressiva. L'incidenza cumulativa di morte renale a 4 anni era infatti pari al $27 \%$ nel gruppo di controllo e al $10 \%$ 
nel gruppo trattato con dieta ipoproteica. Inoltre lo studio Steno dimostra chiaramente un effetto di potenziamento della dieta rispetto ai farmaci (9).

\section{Dieta ipoproteica: aspetti di farmacoeconomia}

Le linee guida internazionali suggeriscono di iniziare il trattamento ipoproteico quando la velocità di filtrazione glomerulare è inferiore a $60 \mathrm{ml} / \mathrm{min}$, ovvero a partire dallo stadio 3 della classificazione NKF-DOQI (4-10). Come si è detto sopra, su 18 pazienti trattati per un anno con la dieta, uno evita il ricorso alla dialisi (3). Se ne conclude che l'utilizzo della dieta può consentire un risparmio per il sistema sanitario. Il costo del trattamento dialitico è di circa $35.000 € /$ anno (49.000 € se si includono i costi sociali), il costo di una dieta con alimenti ipoproteici è di circa $1440 € /$ anno (considerato un consumo di $8 \mathrm{~kg} /$ mese di prodotti aproteici). Su una popolazione di 5000 pazienti si risparmierebbero circa 2.500.000 $€ /$ anno (6.400.000 €/anno includendo i costi sociali) (8).

\section{Dieta ipoproteica: promozione della concordance}

La promozione della compliance è la sfida più importante per il dietista impegnato nel processo assistenziale della nutrizione dei pazienti con IRC (11) e inizia con la considerazione sistematica dei limiti del piano dietetico proposto. Durante la diagnosi nutrizionale, lo sviluppo del piano di trattamento e il follow-up nutrizionale, è necessario valutare attentamente i problemi specifici di ciascun paziente (13), al fine di rendere la relazione dietista-paziente il più efficace possibile soprattutto a lungo termine.

I principali aspetti critici da considerare sono:

1) riduzione o eliminazione degli alimenti di consumo abituale;

2) riduzione della scelta di alimenti;

3) introduzione di nuovi alimenti;

4) introduzione di prodotti ipoproteici;

5) difficoltà di integrazione della cucina tradizionale con quella dietetica;

6) difficoltà di integrazione della vita professionale e di relazione con la terapia dietetica.

Un problema particolare è rappresentato dall'introduzione dei prodotti dietetici ipoproteici. L'impiego di questi prodotti è condizionato principalmente dal tenore proteico della dieta (sempre necessario per valori $\leq 0,6 \mathrm{~g} / \mathrm{kg}$ peso ideale, dal contenuto di proteine ad alto valore biologico (sempre necessario per valori $\geq 75 \%$ ), dal tenore energetico della dieta (sempre necessario per diete ad elevato contenuto di energia) e dalla presenza di comorbidità (per es., nefropatia diabetica) (13). La concordance è influenzata anche dalla qualità percepita degli alimenti ipoproteici e dalla loro disponibilità locale (14).

\section{Dieta ipoproteica: aspetti di politica sanitaria}

Il Servizio Sanitario Nazionale ha normato l'erogazione degli alimenti ipoproteici con il DM 8/6/2001 “Assistenza sanitaria integrativa relativa ai prodotti destinati ad una alimentazione particolare" incluso nel Decreto del Presidente del Consiglio dei Ministri del 29 novembre 2001 "Definizione dei Livelli Essenziali di Assistenza", senza prevedere un criterio omogeneo di erogazione degli alimenti ipoproteici sul territorio nazionale. Ogni Regione identifica differenti criteri di erogazione che rispondono a differenti "decisioni regionali". La Tabella I, elaborata con i dati più aggiornati (settembre 2014), mostra tale variabilità (15).

La disomogeneità dei criteri di erogazione sul territorio nazionale così come la limitazione dettata dal frazionamento merceologico del tetto di spesa (in Veneto e Umbria si individuano a priori le categorie di prodotti ipoproteici) condizionano l'attuazione del trattamento dietetico, influenzando l'efficacia della terapia dietetica e la compliance/concordance. Inoltre la ridotta offerta dei prodotti (disponibilità solo di alcuni marchi di pane o pasta, biscotti ecc.) e la loro indisponibilità all'estero, rappresentano ulteriori ostacoli alla promozione della compliance/concordance. In linea generale, il modello di erogazione degli alimenti ipoproteici può rendere più complesso l'approvvigionamento e condizionare la qualità di vita dei pazienti, in particolare di quelli anziani.

\section{Metodi}

Lo studio descritto in questo articolo si proponeva di valutare il gradimento dei prodotti dietetici ipoproteici da parte di un gruppo di pazienti con IRC seguiti nel triennio 2009-2012 e confrontarlo con quelli di un gruppo analogo di pazienti studiato nell'anno 2008-2009.

Abbiamo effettuato uno studio trasversale su 100 pazienti con IRC afferenti all'Ambulatorio di Dietetica delle Malattie Renali del Nuovo Ospedale Civile S. Agostino-Estense, Azienda USL Modena, nel periodo fra settembre 2009 e giugno 2012. I pazienti eleggibili per lo studio potevano essere in trattamento conservativo con impiego attuale di alimenti ipoproteici o in trattamento sostitutivo con impiego pregresso recente di tali alimenti.

È stato utilizzato un questionario composto di 16 domande chiuse e di 1 domanda aperta compilato da un dietista tramite intervista telefonica. Le variabili ordinali sono state classificate con scala Likert da 1 (peggiore o meno frequente) a 5 (maggiore o più frequente) (Tab. II).

\section{Risultati}

\section{Caratteristiche dei pazienti}

I 100 pazienti consecutivi avevano un'età mediana di 65 anni (intervallo da 17 a 88 anni) ed erano uomini per il 57\%. Il trattamento in corso era conservativo per $1^{\prime} 88 \%$ dei pazienti e sostitutivo per il 12\% (10\% dialisi e 2\% trapianto). Il $62 \%$ dei pazienti era pensionato, il 13\% lavoratore dipendente, il $10 \%$ libero professionista, il 13\% disoccupato o casalinga, e il $2 \%$ studente. La mediana del numero dei componenti del nucleo familiare era di 2 (intervallo da 1 a 6 ). Tutti i pazienti erano o erano stati in trattamento dietetico con una dieta ipoproteica da 0,6 o $0,8 \mathrm{~g} / \mathrm{kg}$ peso corporeo ideale/die. 
TABELLA I - EROGAZIONE DEI PRODOTTI IPOPROTEICI IN ITALIA

\begin{tabular}{|c|c|c|c|c|}
\hline \multirow{2}{*}{$\begin{array}{l}\text { Regione } \\
\text { Valle } \\
\text { d'Aosta }\end{array}$} & \multirow{2}{*}{ Rimborso } & \multicolumn{2}{|c|}{ Spesa Mensile/Quantità Rimborsata } & \multirow{2}{*}{$\begin{array}{l}\text { Modalità } \\
\text { di Erogazione } \\
\text { Farmacie territoriali* } \\
\text { o negozi specializzati** }\end{array}$} \\
\hline & & 105 euro & & \\
\hline Piemonte & $\checkmark$ & 120 euro & & $\begin{array}{l}\text { Farmacie ospedaliere/territoriali } \\
\text { o negozi specializzati }\end{array}$ \\
\hline Lombardia & $\checkmark$ & Nessun tetto di spesa & & $\begin{array}{l}\text { Farmacie ospedaliere/territoriali } \\
\text { o negozi specializzati in base al } \\
\text { piano dietoterapeutico prescritto } \\
\text { al paziente }\end{array}$ \\
\hline \multirow[t]{2}{*}{$\begin{array}{l}\text { Trentino } \\
\text { Alto Adige }\end{array}$} & $\begin{array}{c}\text { Trento } \\
\checkmark\end{array}$ & & $\begin{array}{l}20 \mathrm{~kg} \text { di prodotti al mese } \\
\text { Il paziente anticipa il costo dei } \\
\text { prodotti. L'ASL*** rimborsa dopo } 3 \\
\text { mesi l' } 80 \% \text { della spesa totale }\end{array}$ & $\begin{array}{l}\text { Farmacie territoriali o negozi } \\
\text { specializzati }\end{array}$ \\
\hline & $\begin{array}{l}\text { Bolzano } \\
x\end{array}$ & & & $\begin{array}{l}\text { Farmacie territoriali o negozi } \\
\text { specializzati }\end{array}$ \\
\hline Veneto & $\checkmark$ & $\begin{array}{l}\mathrm{VFG}<50 \mathrm{ml} / \mathrm{min} \text { : } 100 \text { euro con frazionamento merce- } \\
\text { ologico di pane, pasta, farina e biscotti in base al piano } \\
\text { dietoterapeutico prescritto al paziente }\end{array}$ & & $\begin{array}{l}\text { Farmacie territoriali o negozi } \\
\text { specializzati }\end{array}$ \\
\hline Liguria & $\checkmark$ & $\begin{array}{l}\text { Nessun tetto di spesa. Ritiro dei prodotti in base al piano } \\
\text { dietoterapeutico prescritto al paziente. } \\
\text { Il paziente anticipa il costo dei prodotti; successivamen- } \\
\text { te verrà rimborsato in base alla fascia di reddito a cui } \\
\text { appartiene (presentazione modulo ISEE): } \\
\text { - reddito }<20.000 \text { euro: rimborso totale; } \\
\text { - reddito } 20.000-40.000 \text { euro: } 70 \text { euro; } \\
\text { - reddito }>40.000 \text { euro: importo totale } \\
\text { a carico dell'assistito }\end{array}$ & & $\begin{array}{l}\text { Farmacie territoriali o negozi } \\
\text { specializzati }\end{array}$ \\
\hline \multirow{3}{*}{$\begin{array}{l}\text { Emilia } \\
\text { Romagna }\end{array}$} & $\checkmark$ & VFG $55-25 \mathrm{ml} / \mathrm{min}: 60$ euro & & Farmacie territoriali \\
\hline & & $\mathrm{VFG}<25 \mathrm{ml} / \mathrm{min}: 100$ euro & & \\
\hline & & $\begin{array}{l}\text { REPUBBLICA } \\
\text { DI SAN MARINO }\end{array}$ & $9 \mathrm{~kg}$ di prodotti al mese & $\begin{array}{l}\text { Ritiro/acquisto prodotti nelle } \\
\text { ASL }\end{array}$ \\
\hline Toscana & $\checkmark$ & 50 euro & & $\begin{array}{l}\text { Farmacie ospedaliere/territoriali } \\
\text { o negozi specializzati }\end{array}$ \\
\hline Umbria & $\checkmark$ & $\begin{array}{l}90 \text { euro tetto spesa. Ticket di } 0,50 \text { euro su ogni prodotto } \\
\text { ritirato a carico del paziente }\end{array}$ & & $\begin{array}{l}\text { Farmacie territoriali o negozi } \\
\text { specializzati }\end{array}$ \\
\hline Marche & $\checkmark$ & 41 euro & & $\begin{array}{l}\text { Farmacie territoriali o negozi } \\
\text { specializzati }\end{array}$ \\
\hline Lazio & $\checkmark$ & $\begin{array}{l}\text { Solo per } \mathrm{VFG}<30 \mathrm{ml} / \mathrm{min} \\
\text { - } 120 \text { euro per adulti e bambini di età superiore a } 12 \\
\text { anni; } \\
\text { - } 160 \text { euro per bambini di età inferiore a } 12 \text { anni che } \\
\text { assumono latte aproteico }\end{array}$ & & $\begin{array}{l}\text { Farmacie territoriali o negozi } \\
\text { specializzati }\end{array}$ \\
\hline Abruzzo & $x$ & & & Farmacie ospedaliere \\
\hline Molise & $x$ & & & $\begin{array}{l}\text { Farmacie territoriali e negozi } \\
\text { specializzati }\end{array}$ \\
\hline Campania & $x$ & & & $\begin{array}{l}\text { Farmacie ospedaliere/territoriali } \\
\text { o negozi specializzati }\end{array}$ \\
\hline Puglia & $\checkmark$ & $\begin{array}{l}\text { Nessun tetto di spesa: ritiro prodotti } \\
\text { in base al piano dietoterapeutico prescritto al } \\
\text { paziente }\end{array}$ & & $\begin{array}{l}\text { Farmacie territoriali o negozi } \\
\text { specializzati }\end{array}$ \\
\hline
\end{tabular}


TABELLA I - CONTINUA

\begin{tabular}{|c|c|c|c|c|}
\hline \multirow{2}{*}{$\begin{array}{l}\text { Regione } \\
\text { Basilicata }\end{array}$} & \multirow{2}{*}{$\begin{array}{c}\text { Rimborso } \\
\checkmark\end{array}$} & \multicolumn{2}{|c|}{ Spesa Mensile/Quantità Rimborsata } & \multirow{2}{*}{$\begin{array}{l}\text { Modalità } \\
\text { di Erogazione }\end{array}$} \\
\hline & & $\begin{array}{l}\text { Nessun tetto di spesa: ritiro prodotti } \\
\text { in base al piano dietoterapeutico prescritto al paziente }\end{array}$ & & \\
\hline Calabria & $\checkmark$ & 100 euro & & $\begin{array}{l}\text { Farmacie territoriali o negozi } \\
\text { specializzati }\end{array}$ \\
\hline Sicilia & $\checkmark$ & VFG $<30 \mathrm{ml} / \mathrm{min}: 80$ euro & & $\begin{array}{l}\text { Farmacie ospedaliere/territoriali } \\
\text { o negozi specializzati }\end{array}$ \\
\hline Sardegna & $\checkmark$ & & $\begin{array}{l}\text { Dai } 7 \text { ai } 10 \mathrm{~kg} \text { di prodotti al mese, } \\
\text { in base al piano dietoterapeutico } \\
\text { prescritto al paziente }\end{array}$ & Farmacie ospedaliere \\
\hline
\end{tabular}

$\checkmark$ Presenza di rimborso.

$x$ Assenza di rimborso.

* Farmacie presenti sul territorio.

** Negozi dedicati alla vendita di prodotti senza glutine che richiedono all'ASL di riferimento una specifica autorizzazione per la distribuzione dei prodotti dietetici ipoproteici.

*** Azienda Sanitaria Locale.

VGF: volume di filtrazione glomerulare

TABELLA II - GRADIMENTO PERCENTUALE DEI PRODOTTI IPOPROTEICI VALUTATO CON SCALA LIKERT DA 1 A 5

\begin{tabular}{|c|c|c|c|c|c|}
\hline & Pasta & Pane & Biscotti & Farina & Bevanda \\
\hline 1 scarsa & $5 \%$ & $16 \%$ & $6 \%$ & $5 \%$ & $20 \%$ \\
\hline 2 sufficiente & $9 \%$ & $11 \%$ & $20 \%$ & $12 \%$ & $0 \%$ \\
\hline 3 buona & $23 \%$ & $30 \%$ & $31 \%$ & $53 \%$ & $40 \%$ \\
\hline 4 molto buona & $35 \%$ & $24 \%$ & $12 \%$ & $13 \%$ & $20 \%$ \\
\hline 5 ottima & $28 \%$ & $19 \%$ & $31 \%$ & $17 \%$ & $20 \%$ \\
\hline
\end{tabular}

\section{Risposte al questionario}

Domanda 1: Quali sono i prodotti ipoproteici che consuma?

\begin{tabular}{lr}
\hline Pasta & $92 \%$ \\
Pane o sostituti & $53 \%$ \\
Biscotti o snack & $33 \%$ \\
Farina & $9 \%$ \\
Bevanda & $5 \%$
\end{tabular}

Domanda 2: Qual è la gradibilità rispetto a questi prodotti?

Pasta

$86 \%$

Biscotti o snack $\quad 74 \%$

Pane o sostituti $\quad 73 \%$

Farina

$5 \%$

$20 \%$ ottimo o molto buono molto buono molto buono

scarso

scarso

In Tabella II alleghiamo il dettaglio del gradimento dei prodotti ipoproteici valutato con scala Likert da 1 a 5 .

Domanda 3: Da quanto tempo consuma i prodotti ipoproteici? Da più di 12 mesi $\quad 58 \%$

Tra 6 e 12 mesi $\quad 13 \%$

Tra 3 e 6 mesi $\quad 12 \%$

Tra 1 e 3 mesi $\quad 8 \%$

Da meno di 1 mese $\quad 9 \%$
Domanda 4: In quali pasti consuma i prodotti ipoproteici?

Colazione $\quad 32 \%$

Spuntino $\quad 8 \%$

Pranzo $\quad 89 \%$

Merenda $\quad 12 \%$

Cena $\quad 63 \%$

Domanda 5: Quali pasti consuma abitualmente a casa?

$\begin{array}{ll}\text { Colazione } & 72 \% \\ \text { Spuntino } & 22 \% \\ \text { Pranzo } & 91 \% \\ \text { Merenda } & 33 \% \\ \text { Cena } & 92 \%\end{array}$

Domanda 6: Chi prepara i pasti a casa?

\begin{tabular}{lr}
\hline Io stesso & $57 \%$ \\
Partner & $43 \%$ \\
Genitore & $3 \%$ \\
Figlio/nipote & $3 \%$ \\
Collaboratrice domestica & $0 \%$
\end{tabular}

Domanda 7: Come sono le abilità culinarie di chi prepara i pasti?

$\begin{array}{ll}\text { Ottime } & 36 \% \\ \text { Molto buone } & 25 \% \\ \text { Buone } & 28 \%\end{array}$


Accettabili $\quad 7 \%$

Scarse $\quad 4 \%$

Domanda 8: Le capita di impiegare i prodotti ipoproteici per la preparazione di ricette regionali (per es., lasagne, tigelle, tortellini, focaccia, pizza, piadina, pasta al forno, besciamella, crepes, torte)?

$\begin{array}{lr}\text { Abitualmente } & 2 \% \\ \text { Spesso } & 1 \% \\ \text { Qualche volta } & 9 \% \\ \text { Raramente } & 15 \% \\ \text { Mai } & 73 \%\end{array}$

Domanda 9: Se ha risposto affermativamente alla Domanda 8 , indichi le preparazioni che realizza solitamente con i prodotti ipoproteici (specificando i prodotti ipoproteici impiegati) Pizza o tigelle $\quad 8 \%$

Lasagne $\quad 6 \%$

Torte $\quad 5 \%$

Crema $\quad 3 \%$

Pane o focaccia $2 \%$

Besciamella $\quad 1 \%$

Pasta al forno $\quad 1 \%$

NB: Le risposte in percentuale sono riferite all'intero campione.

Domanda 10: Se ha risposto affermativamente alla Domanda 8, quali sono i risultati dell'uso dei prodotti ipoproteici nella preparazione di queste ricette?

$\begin{array}{ll}\text { Ottimi } & 10 \% \\ \text { Molto buoni } & 20 \% \\ \text { Buoni } & 20 \% \\ \text { Accettabili } & 10 \% \\ \text { Scarsi } & 40 \%\end{array}$

Domanda 11: Le preparazioni realizzate con i prodotti ipoproteici sono differenti rispetto a quelle realizzate con $\mathrm{i}$ prodotti "comuni"?

$\begin{array}{ll}\text { Moltissimo } & 13 \% \\ \text { Molto } & 22 \% \\ \text { Abbastanza } & 35 \% \\ \text { Poco } & 18 \% \\ \text { Per niente } & 12 \%\end{array}$

Domanda 12: I metodi di preparazione dei piatti a contenuto proteico controllato sono più impegnativi rispetto a quelli dei piatti comuni?

$\begin{array}{lr}\text { Moltissimo } & 3 \% \\ \text { Molto } & 10 \% \\ \text { Abbastanza } & 8 \% \\ \text { Poco } & 5 \% \\ \text { Per niente } & 74 \%\end{array}$

Domanda 13: I tempi di preparazione dei piatti a ridotto contenuto proteico sono più lunghi rispetto a quelli dei piatti comuni?

$\begin{array}{ll}\text { Moltissimo } & 1 \% \\ \text { Molto } & 6 \% \\ \text { Abbastanza } & 3 \%\end{array}$

$\begin{array}{ll}\text { Poco } & 13 \% \\ \text { Per niente } & 77 \%\end{array}$

Domanda 14: A suo parere, i prodotti ipoproteici sono validi sostituti dei prodotti comuni?

$\begin{array}{lr}\text { Molto validi } & 36 \% \\ \text { Validi } & 34 \% \\ \text { Abbastanza validi } & 24 \% \\ \text { Poco validi } & 4 \% \\ \text { Per niente validi } & 2 \%\end{array}$

Domanda 15: I prodotti ipoproteici vengono consumati anche dai suoi familiari?

$\begin{array}{lr}\text { Sempre } & 6 \% \\ \text { Spesso } & 2 \% \\ \text { Qualche volta } & 17 \% \\ \text { Raramente } & 19 \% \\ \text { Mai } & 56 \%\end{array}$

Domanda 16: Ritiene che il fabbisogno mensile dei prodotti ipoproteici erogato dalla sua Regione sia adeguato alle sue necessità?

$\begin{array}{lr}\text { Moltissimo } & 66 \% \\ \text { Molto } & 18 \% \\ \text { Abbastanza } & 13 \% \\ \text { Poco } & 1 \% \\ \text { Per niente } & 2 \% *\end{array}$

*pazienti che non risiedono in Emilia Romagna

Domanda 17: Indichi eventuali suggerimenti per migliorare i prodotti ipoproteici.

A questa domanda ha risposto il 33\% dei pazienti. Di seguito riportiamo i principali suggerimenti:

1) migliorare la tecnologia di produzione della pasta in modo da garantire una migliore tenuta in cottura $(18 \%)$;

2) aumentare la varietà dei prodotti (per es., piatti pronti. Alcuni lamentano la sospensione della produzione di alcune tipologie di alimenti rispetto al passato) (15\%);

3) migliorare la consistenza del pane (riferita come "gommosa") (15\%);

4) ridurre il gusto troppo dolce di biscotti e snack dolci (9\%);

5) migliorare la sapidità degli alimenti (9\%);

6) fornire maggiori informazioni sui metodi di preparazione degli alimenti (9\%);

7) rendere più morbide le fette biscottate $(6 \%)$;

8) migliorare il colore dei prodotti $(6 \%)$;

9) utilizzare confezioni meno ingombranti (3\%);

10) utilizzare adesivi per richiudere le confezioni (3\%);

11) fornire pane pronto al consumo senza bisogno di essere riscaldato $(3 \%)$.

\section{Discussione}

In linea con lo studio precedente effettuato nell'anno 20082009 (16), la pasta si conferma l'alimento ipoproteico maggiormente consumato ma si assiste a un incremento del consumo di pane e sostituti con associato maggiore gradimento, verosimilmente attribuibile al miglioramento delle tecno- 
logie produttive. La durata media di consumo dei prodotti aproteici e la loro distribuzione nella giornata sono sovrapponibili tra i due studi. La preparazione di piatti regionali con alimenti ipoproteici è più frequente per i dolci, infine la differenza di gusto tra piatti comuni e a contenuto proteico controllato viene percepita maggiore nel presente studio.

\section{Conclusioni}

Nonostante la qualità dei prodotti ipoproteici sia nettamente migliorata nel corso degli anni, non vi è dubbio che il loro inserimento nel modello alimentare sia uno dei fattori che condiziona maggiormente la compliance/concordance dei pazienti (16). La prescrizione di un prodotto ipoproteico dovrebbe essere sempre accompagnata dalla spiegazione del suo razionale di utilizzo e delle sue modalità di preparazione e, quando possibile, da un "taste-test" preliminare, utile per favorire la collaborazione e il coinvolgimento di pazienti e familiari, modalità che nel nostro Ambulatorio è prevista per tutti i pazienti che iniziano un trattamento nutrizionale in cui sia prevista l'introduzione degli alimenti ipoproteici.

\section{Riassunto}

Il supporto nutrizionale del paziente con Insufficienza Renale Cronica (IRC) in trattamento conservativo consente di prevenire/controllare le principali alterazioni metaboliche della malattia, raggiungere e/o mantenere uno stato nutrizionale soddisfacente posticipando il trattamento sostitutivo (dialisi o trapianto).

Quando la dieta ipoproteica richiede l'introduzione di alimenti speciali ipoproteici, il dietista deve effettuare un monitoraggio particolarmente attento.

Il presente studio ha valutato il gradimento degli alimenti ipoproteici in un campione di 100 pazienti con IRC afferenti alla nostra Unità Operativa di Scienza dell'Alimentazione nel triennio 2009-2012 e lo ha confrontato con quello riscontrato in uno studio simile effettuato nell'anno 20082009. L'età mediana del campione era 65 anni (intervallo da 17 a 88 anni), con prevalenza del sesso maschile (57\%). L' $88 \%$ dei pazienti era in trattamento conservativo e il $12 \%$ in trattamento sostitutivo ( $10 \%$ dialisi e $2 \%$ trapianto). Tutti i pazienti erano stati trattati con una dieta ipoproteica con 0.6 o $0.8 \mathrm{~g}$ proteine / $\mathrm{kg}$ peso corporeo ideale / die.

Soltanto il 6\% dei pazienti dichiarava "nulla" o "scarsa" la sostituibilità dei prodotti normali con quelli ipoproteici. Rispetto allo studio precedente, si è assistito a un incremento del consumo di pane e sostituti con associato maggiore gradimento. Anche se la varietà e la qualità dei prodotti ipoproteici sono nettamente aumentate nel corso degli anni, il loro inserimento nel modello alimentare è uno dei fattori che condizionano maggiormente la concordance al trattamento dietetico.

In relazione alle esigenze della nostra utenza, abbiamo affiancato allo studio una revisione delle normative regionali inerenti l'erogazione dei prodotti ipoproteici, che evidenzia una marcata disomogeneità sul territorio nazionale.

Parole chiave: Dieta ipoproteica, Prodotti ipoproteici, Insufficienza renale cronica, Studio trasversale

Dichiarazione di conflitto di interesse: Gli Autori dichiarano di non avere conflitto di interessi.

Contributi economici agli Autori: Gli Autori dichiarano di non avere ricevuto sponsorizzazioni economiche per la preparazione dell'articolo.

Indirizzo degli Autori:

Dr.ssa Anna Laura Fantuzzi

Dietista Coordinatore

Unità Operativa Dipartimentale di Scienza dell'Alimentazione e Dietetica

Azienda USL di Modena

Nuovo Ospedale Civile S. Agostino-Estense

Via Giardini 1355

41126 Modena

a.fantuzzi@ausl.mo.it

\section{Bibliografia}

1. Fouque D. Why is the diet intervention so critical during chronic kidney disease? J Ren Nutr 2003; 13: 173.

2. Wiggins KL. Guidelines for nutritional care of renal patients. Chicago: Renal Dietitians Dietetic Practice Group of the American Dietetic Association, 2003.

3. Fouque D, Laville M. Low protein diets for chronic kidney disease in non diabetic adults. Cochrane Database Syst Rev 2009: CD001892.
4. Cianciaruso B, Bellizzi V, Brunori G, et al. Low-protein dietary therapy in patients with chronic kidney disease. G Ital Nefrol 2008; 25(Suppl 42): S1-S2.

5. Cianciaruso B, Bellizzi V, Brunori G, et al. Low-protein diet in Italy today: the conclusions of the Working Group from the Italian Society of Nephrology. G Ital Nefrol 2008; 25(Suppl 42): S54-S57.

6. Fouque D, Aparicio M. Eleven reasons to control the protein intake of patients with chronic kidney disease. Nat Clin Pract Nephrol 2007; 3: 383-92. 
7. Wiggins KL, Harvey KS. A review of guidelines for nutrition care of renal patients. J Ren Nutr 2002; 12: 190-6.

8. Williams ME, Chianchiano D. Medicare medical nutrition therapy: legislative process and product. J Ren Nutr 2002; 12: 1-7.

9. Hansen HP, Tauber-Lassen E, Jensen BR, Parving HH. Effect of dietary protein restriction on prognosis in patients with diabetic nephropathy. Kidney Int 2002; 62: 220-8.

10. Cupisti A. Definition of the nutritional therapy in the conservative treatment of chronic kidney disease. G Ital Nefrol 2008; 25(Suppl 42): S35-S38.

11. Writing Group of the Nutrition Care Process/standardized Language Committee. J Am Diet Assoc 2008; 108: 1113-7.

12. Quintaliani G, Cappelli G, Lodetti L, et al. Chronic kidney disease certification process manual by the Italian Society of Nephrology (SIN): Part I: clinical care delivery and performance measurements and improvement. J Nephrol 2009: 22(4): 423-38.

13. Fantuzzi AL, Bedogni G. Dieta ipoproteica e insufficienza renale cronica: manuale pratico per il Dietista. Milano: UTET, 2003.

14. Bellizzi V, Bedogni G, Quintaliani G. Compliance with low protein diet in patients with cronic kidney disease. G Ital Nefrol 2008; 25(Suppl. 42): S45-S49.

15. www.renalgate.it/nutrizione

16. Fantuzzi AL, Giannini R, Bedogni G. Indagine sull'impiego dei prodotti dietetici ipoproteici nei pazienti con insufficienza renale cronica. La rivista di Scienza dell'Alimentazione 2010; 39(3): 21-6. 